\title{
२UALITATIVE THEORY OF PARTIAL DIFFERENCE EQUATIONS (V): STURMIAN THEOREMS FOR A CLASS OF PARTIAL DIFFERENCE EQUATIONS
}

\author{
SUI SUN CHENG*, SHENG-LI XIE AND BING GEN ZHANG ${ }^{\dagger}$
}

\begin{abstract}
This paper is concerned with Sturmian theorems for a class of partial difference equations. These results are obtianed by means of averaging techniques, variational inequalities and comparison theorems.
\end{abstract}

\section{Introduction}

Oscillation criteria for several classes of partial difference equations have been derived in [2-5]. In this paper, we will be concerned with Sturmian theorems for a class of partial difference equations of the form

$$
\Delta_{2}\left(A(j-1) \Delta_{2} u(i, j-1)\right)+B(j) \Delta_{1}^{2} u(i-1, j)+C(j) u(i, j)=0
$$

where $1 \leq i \leq n$, and $j \geq 1$, and $A(j)>0$ for $j \geq 0$. When $B(j)>0$ for $j \geq 1$, this equation can be regarded as a discrete analog of an elliptic equation, while if $B(j)<0$ for $j \geq 1$, it can be regarded as a discrete analog of a hyperbolic equation.

Let $\alpha, \beta \geq 0$. We will be interested in the existence of a rectangle of the form

$$
[1, n] \times\left[s-\frac{1}{1+\alpha}, t+\frac{1}{1+\beta}\right]
$$

such that no solution $u(i, j)$ of $(1.1)$ "can remain positive" over this rectangle. To be more precise, let $f(i)$ be defined on a set of consecutive integers $a, a+1, \ldots, b$. If the

Received November 22, 1994; revised April 20, 1995.

1991 Mathematics Subject Classification. 39A10, 35R10.

Key words and phrases. Partial difference equations, Sturmian theorems.

* Partially supported by National Science Council of R.O.C.

$\uparrow$ Partially supported by NNSF of China. 
points $(i, f(i)), a \leq i \leq b$, are jointed by straight line segments to form a broken line, then this broken line gives rise to a representation of a continuous function, called the linear interpolant of $f$ and denoted by $f^{*}$, such that $f^{*}(k)=f(k)$ for $k=a, \ldots, b$. We say that $f$ remains positive over a real interval $I$ if its linear interpolant $f^{*}$ is positive over $I$. Similarly, let $f(i, j)$ be defined on a set of lattice points of the form

$$
\{(i, j) \mid i=a, a+1, \ldots, b ; j=c, c+1, \ldots, d ; a, b, c, d \text { are integers }\}
$$

For each fixed $j_{0}$ in $\{c, \ldots, d\}$, we join the points $\left(i, f\left(i, j_{0}\right)\right), a \leq i \leq b$, by straight line segments, and for each fixed $i_{0}$ in $\{a, \ldots, b\}$, we join the points $\left(j, f\left(i_{0}, j\right)\right), c \leq$ $j \leq d$, by straight line segments to form a broken net. Again, we denote the function which represents this net by $f^{*}$, and say that $f(i, j)$ is positive over a real rectangle $\Gamma$ if $f^{*}(s, t)>0$ for $(s, t)$ in the intersection of $\Gamma$ and the domain of $f^{*}$.

Such a question described above is similar to the classical problem of the existence of an interval $I$ such that any solution of a Sturm-Liouville equation has a zero in this interval. As is well known, the Sturm-Picone comparison theorem for such an equation makes use of a minorant equation and two consecutive zeros $\nu, \lambda$ of one of its solution to provide such an interval $[\nu, \lambda]$, while Leighton's variational lemma [8] makes use of a quadratic functional for the same purpose.

Here we will employ similar ideas and consider a functional of the form

$$
\sum_{k=s}^{t-1} A(k)(\Delta y(k))^{2}-\sum_{k=s}^{t}(C(k)-\mu B(k)) y^{2}(k)+(1+\alpha) A(s-1) y^{2}(s)+(1+\beta) A(t) y^{2}(t),
$$

where $\mu$ is to be specified later, and a minorant partial difference equation of the form

$$
\Delta_{2}\left(a(j-1) \Delta_{2} v(i, j-1)\right)+b(j) \Delta_{1}^{2} v(i-1, j)+c(j) v(i, j)=0
$$

In place of the interval $[\nu, \lambda]$, we consider a rectangle of the form

$$
[0, n+1] \times[s-1 /(\alpha+1), t+1 /(\beta+1)]
$$

such that (1.2) has a solution $v(i, j)$ which satisfies the conditions

$$
\begin{gathered}
v(0, j)=0=v(n+1, j), \quad s \leq j \leq t \\
v(i, s-1)+\alpha v(i, s)=0=v(i, t+1)+\beta v(i, t), \quad 1 \leq i \leq n . \\
v(i, j)>0, \quad 1 \leq i \leq n, s \leq j \leq t .
\end{gathered}
$$

For the sake of convenience, we shall call such a rectangle a rectangular nodal domain of (1.2). We assert that if (1.1) majorizes (1.2) in an appropriate manner, then no solution of (1.1) can remain positive nor negative over any rectangular nodal domain of (1.2). 


\section{Preparatory Lemmas}

In order to derive the assertion made in the previous Section, we shall need the following two preparatory results which have been stated and used in our previous works $[3,4]$ for reducing partial difference equations to ordinary recurrence relations.

Lemma 2.1. Let

$$
g(i, j)= \begin{cases}(n-i+1) j /(n+1) & 1 \leq j \leq i \leq n \\ (n-j+1) i /(n+1) & 1 \leq i \leq j \leq n\end{cases}
$$

Then $g(i, j)$ is symmetric, positive for $1 \leq i, j \leq n$ and

$$
\sum_{j=1}^{n} g(i, j) \Delta^{2} v(j-1)=-v(i)+\delta(i), \quad 1 \leq i \leq n
$$

where $\delta(1)=n v(0) /(n+1), \delta(n)=n v(n+1) /(n+1)$ and $\delta(i)=0$ for $2 \leq i \leq n-1$.

Lemma 2.2. The eigenvalue problem

$$
\begin{gathered}
\Delta^{2} w(k-1)+\lambda w(k)=0, \quad 1 \leq k \leq n, \\
w(0)=0=w(n+1),
\end{gathered}
$$

is equivalent to the symmetric eigenvalue problem

$$
w(i)=\lambda \sum_{j=1}^{n} g(i, j) w(j), \quad 1 \leq i \leq n
$$

which possesses the positive eigenvalue

$$
\mu_{n}=4 \sin ^{2}\left(\frac{\pi}{2 n+2}\right) .
$$

This eigenvalue is smaller than all other eigenvalues and its corresponding eigensolution $w(k)$ is given by

$$
w(k)= \begin{cases}0 & k=0 \text { or } n+1 \\ \sin (k \pi /(n+1)) & 1 \leq k \leq n\end{cases}
$$

Now let $\{u(i, j)\}$ be a solution of (1.1). Let

$$
U(j)=\sum_{i=1}^{n} u(i, j) w(i), \quad j \geq 0,
$$


where $w(j)$ is defined by (2.5). Then multiplying (1.1) by $w(i)$ and then summing from $i=1$ to $i=n$, we have

$$
\Delta(A(j-1) \Delta U(j-1))+B(j) \sum_{i=1}^{n} w(i) \Delta_{1}^{2} u(i-1, j)+C(j) U(j)=0, \quad j \geq 1 .
$$

In view of Lemma 2.1 ,

$$
\begin{aligned}
& \sum_{i=1}^{n} w(i) \Delta_{1}^{2} u(i-1, j)=\sum_{i=1}^{n}\left\{\mu_{n} \sum_{s=1}^{n} g(s, i) w(s) \Delta_{1}^{2} u(i-1, j)\right\} \\
= & \mu_{n} \sum_{s=1}^{n}\left\{\sum_{i=1}^{n} g(s, i) \Delta_{1}^{2} u(i-1, j)\right\} w(s)=\mu_{n} \sum_{s=1}^{n}\left\{-u(s, j)+\delta_{j}(s)\right\} w(s) \\
= & -\mu_{n} U(j)+\mu_{n} \sum_{s=1}^{n} \delta_{j}(s) w(s)=-\mu_{n} U(j)+\delta_{j}(1) \mu_{n} w(1)+\delta_{j}(n) \mu_{n} w(n) \\
= & -\mu_{n} U(j)+\frac{n}{n+1} \mu_{n}\{u(0, j) w(1)+u(n+1, j) w(n)\},
\end{aligned}
$$

thus we obtain

$$
\begin{aligned}
& \Delta(A(j-1) \Delta U(j-1))+\left(C(j)-\mu_{n} B(j)\right) U(j) \\
= & -\frac{n}{n+1} \mu_{n}\{u(0, j) w(1)+u(n+1, j) w(n)\}, \quad j \geq 1 .
\end{aligned}
$$

The following two results are now clear.

Lemma 2.3. Let $\{u(i, j)\}$ be a solution of (1.1) satisfying the boundary conditions

$$
u(0, j)=0, u(n+1, j)=0, \quad s \leq j \leq t,
$$

then the function $\{U(j)\}$ defined by (2.8) satisfies

$$
\Delta(A(j-1) \Delta U(j-1))+\left(C(j)-\mu_{n} B(j)\right) U(j)=0
$$

for $s \leq j \leq t$.

Lemma 2.4. If $[1, m] \times[s-1 /(\alpha+1), t+1 /(\beta+1)]$ is a rectangular nodal domain of (1.2), then the following equation

$$
\Delta(a(j-1) \Delta V(j-1))+\left(c(j)-\mu_{m} b(j)\right) V(j)=0, \quad s \leq j \leq t
$$

has a solution $\{V(j)\}$ which satisfies

$$
V(j)>0, \quad s \leq j \leq t,
$$


and

$$
V(s-1)+\alpha V(s)=0=V(t+1)+\beta V(t)
$$

\section{Sturmian Theorems}

In the last Section, we encounter recurrence relations of the form

$$
\Delta(p(k-1) \Delta u(k-1))+q(k) u(k)=0, \quad k=1,2, \ldots,
$$

where $p(k)>0$ for $k \geq 0$. Several Sturmian theorems have already been established for such relations. The ones which are useful for our purposes will be summarized as follows [1].

Let $\alpha$ and $\beta$ be any two fixed non-negative numbers, and $s, t$ be integers such that $s \leq t$. A vector $u=(u(s-1), u(s), \ldots, u(t+1))$ is said to be admissible if it is nontrivial and satisfies

$$
u(s-1)+\alpha u(s)=0, \quad u(t+1)+\beta u(t)=0 .
$$

For any vector $u=(u(s-1), \ldots, u(t+1))$, we define the functional

$$
J[u \mid p, q]=\sum_{k=s}^{t-1} p(k)(\Delta u(k))^{2}-\sum_{k=s}^{t} q(k) u^{2}(k)+(1+\alpha) p(s-1) u^{2}(s)+(1+\beta) p(t) u^{2}(t)
$$

It is not difficult to see that if $u=(u(s-1), \ldots, u(t+1))$ is an admissible solution of (3.1), then $J[u \mid p, q]=0$. Furthermore, we have the following result.

Lemma 3.1. Suppose $u=(u(s-1), \ldots, u(t+1))$ is a solution of (3.1) for $s-1 \leq k \leq t+1$, and $y=(y(s-1), \ldots, y(t+1))$ is any admissible vector. Then the following hold: (i) if $u$ is admissible, then $J[u \mid p, q]=0$; (ii) if $u$ is admissible and $u(k)>0$ for $s \leq k \leq t$, then $J[y \mid p, q] \geq 0$, moreover, $J[y \mid p, q]=0$ if and only if $y$ is a constant multiple of $u$; and (iii) if $u(k)>0$ for $s \leq k \leq t$, and $u(s-1)+\alpha u(s) \geq 0$ and $u(t+1)+\beta u(t) \geq 0$ with at least one strict inequality, then $J[y \mid p, q]>0$.

As a consequence, if there is a nontrivial vector $y=(y(s-1), y(s), \ldots, y(t+1))$ such that

$$
y(s-1)+\alpha y(s)=0=y(t+1)+\beta y(t)
$$

and

$$
\begin{aligned}
J\left[y \mid A, C-\mu_{n} B, s, t\right]= & \sum_{k=s}^{t-1} A(k)(\Delta y(k))^{2}-\sum_{k=s}^{t}\left(C(k)-\mu_{n} B(k)\right) y^{2}(k) \\
& +(1+\alpha) A(s-1) y^{2}(s)+(1+\beta) A(t) y^{2}(t) \leq 0
\end{aligned}
$$


then no solution of (2.9) can remain positive over the real interval $[s-1 /(1+\alpha), t+$ $1 /(1+\beta)$ ] (more precisely, no solution of $(2.9)$ can remain over the real interval $(s-$ $1 /(1+\alpha), t+1 /(1+\beta))$, unless it is a constant multiple of $y$.)

Accordingly, if there is a vector $y=(y(s-1), y(s), \ldots, y(t+1))$ such that (3.3) and (3.4) are satisfied, then (1.1) cannot have a solution $\{u(i, j)\}$ which satisfies $u(0, j)=$ $0=u(n+1, j)$ for $s \leq j \leq t$, and remains positive over the rectangle $[1, n] \times[s-1 /(1+$ $\alpha), t+1 /(1+\beta)$ ], for otherwise the function $U(j)$ defined by $(2.8)$, in view of Lemma 2.3, would be positive over the rectangle $[s-1 /(1+\alpha), t+1 /(1+\beta)]$.

Theorem 3.1. If there is a nontrivial vector $y=(y(s-1), y(s), \ldots, y(t+1))$ such that (3.3) and (3.4) are satisfied, then (1.1) cannot have a solution $\{u(i, j)\}$ which satisfies $u(0, j)=0=u(n+1, j)$ for $s \leq j \leq t$ and also remains positive over the rectangle $[1, n] \times[s-1 /(1+\alpha), t+1 /(1+\beta)]$.

There now arises the question when the hypotheses of Theorem 3.1 are satisfied. For this purpose, we will take (1.2) as a minorant equation and suppose $[1, m] \times[s-1 /(\alpha+1)$, $t+1 /(\beta+1)]$ is an associated rectangular nodal domain. According to Lemma $2.4,(2.10)$ has a solution $\{V(j)\}$ which satisfies $V(j)>0$ for $s \leq j \leq t$ and

$$
V(s-1)+\alpha V(s)=0=V(t+1)+\beta V(t) .
$$

In view of Lemma 3.1, $J\left[V \mid a(k), b(k)-\mu_{m} c(k), s, t\right]=0$. Furthermore, if we impose the conditions

$$
A(j) \leq a(j), \quad s-1 \leq j \leq t,
$$

and

$$
C(j)-\mu_{n} B(j) \geq c(j)-\mu_{m} b(j), \quad s \leq j \leq t,
$$

then

$$
\begin{aligned}
& J\left[V \mid A, C-\mu_{n} B, s, t\right]=J\left[V \mid A, C-\mu_{n} B, s, t\right]-J\left[V \mid a, c-\mu_{m} b, s, t\right] \\
= & \sum_{k=s}^{t-1}(A(k)-a(k))(\Delta V(k))^{2}-\sum_{k=s}^{t}\left(C(k)-\mu_{n} B(k)-c(k)+\mu_{m} b(k)\right) V^{2}(k) \\
& (A(s-1)-a(s-1))(1+\alpha) V^{2}(s)+(A(t)-a(t))(1+\beta) V^{2}(t) \leq 0 .
\end{aligned}
$$

The following result now follows from Theorem 3.1.

Theorem 3.2. Suppose $[1, m] \times[s-1 /(\alpha+1), t+1 /(\beta+1)]$ is a rectangular nodal domain of (1.2), then under the additional conditions (3.5) and (3.6), (1.1) cannot have a solution $\{u(i, j)\}$ which satisfies $u(0, j)=0=u(n+1, j)$ for $s \leq j \leq t$ and also remains positive over the rectangle $[1, n] \times[s-1 /(1+\alpha), t+1 /(1+\beta)]$.

There are at least two variants of Theorem 3.2 which we can obtain without too much efforts. First, instead of the partial difference equation (1.2), we may consider 
a minorant equation of the form (3.1). By means of the same reasoning used in the derivation of Theorem 3.2, we obtain the following result.

Theorem 3.3. Suppose (3.1) has a solution $\{x(k)\}$ which satisfies $x(k)>0$ for $s \leq k \leq t$ and

$$
x(s-1)+\alpha x(s)=0=x(t+1)+\beta x(t) .
$$

Suppose further that

$$
A(k) \leq p(k), \quad s-1 \leq k \leq t,
$$

and

$$
C(k)-\mu_{n} B(k) \geq q(k), \quad s \leq k \leq t .
$$

Then (1.1) cannot have a solution $\{u(i, j)\}$ which satisfies $u(0, j)=0=u(n+1, j)$ for $s \leq j \leq t$ and also remains positive over the rectangle $[1, n] \times[s-1 /(1+\alpha), t+$ $1 /(1+\beta)]$.

Next, we suppose (3.1) has a non-trivial solution $\{x(k)\}$ which satisfies (3.7). Denoting $C(k)-\mu_{n} B(k)$ by $Q(k)$, we assert that if

$$
\sum_{k=s}^{t}\left\{\left(Q(k)-\frac{A(k-1) q(k)}{p(k-1)}\right) x^{2}(k)+p(k) x(k) \Delta x(k) \Delta\left(\frac{A(k-1)}{p(k-1)}\right)\right\} \geq 0
$$

then (1.1) cannot have a solution $\{u(i, j)\}$ which remains positive (or remains negative) for $1 \leq i \leq n$ and $s \leq j \leq t$. Indeed, note that

$$
\begin{aligned}
& \Delta(A(k-1) \Delta x(k-1))+Q(k) x(k) \\
= & \Delta\left\{\frac{A(k-1)}{p(k-1)} p(k-1) \Delta x(k-1)\right\}+Q(k) x(k) \\
= & \left(\frac{A(k-1)}{p(k-1)}\right) \Delta(p(k-1) \Delta x(k-1))+p(k) \Delta x(k) \Delta\left(\frac{A(k-1)}{p(k-1)}\right)+Q(k) x(k) \\
= & \left(Q(k)-\frac{A(k-1) q(k)}{p(k-1)}\right) x(k)+p(k) \Delta u(k) \Delta\left(\frac{A(k-1)}{p(k-1)}\right) .
\end{aligned}
$$

If we multiply the left hand side of (3.9) by $x(k)$ and rearrange to obtain

$$
\Delta(A(k-1) x(k-1) \Delta x(k-1))-A(k-1)(\Delta x(k-1))^{2}+Q(k) x^{2}(k)
$$

then after summing from $k=s$ to $k=t$, we will have

$$
A(t) x(t) \Delta x(t)-A(s-1) x(s-1) \Delta x(s-1)-\sum_{k=s-1}^{t-1} A(k)(\Delta x(k))^{2}+\sum_{k=s}^{t} Q(k) x^{2}(k) .
$$

If we now substitute $\Delta x(t)=-(1+\beta) x(t)$ and $\Delta x(s-1)=(1+\alpha) x(s)$ into the above formula, we obtain

$$
-J[x \mid A, Q, s, t] .
$$


On the other hand, if we multiply the right hand side of (3.9) by $x(k)$ and then sum from $k=s$ to $k=t$, we obtain the left hand side of (3.8). Our assertion thus follows from Theorem 3.1.

Theorem 3.4. Suppose (3.1) has a solution $\{x(k)\}$ which satisfies (3.7). If (3.8) is satisfied, where $Q(k)=C(k)-\mu_{n} B(k)$, then (1.1) cannot have a solution $\{u(i, j)\}$ which satisfies $u(0, j)=0=u(n+1, j)$ for $s \leq j \leq t$ and also remains positive over the rectangle $[1, n] \times[s-1 /(1+\alpha), t+1 /(1+\beta)]$.

\section{An Example}

Let us say that a solution $\{u(i, j)\}$ of the boundary problem

$$
\begin{gathered}
u(0, j)=0=u(n+1, j), \quad j \geq 0 \\
\Delta_{2}\left(A(j-1) \Delta_{2} u(i, j-1)\right)+B(j) \Delta_{1}^{2} u(i-1, j)+C(j) u(i, j)=0,1 \leq i \leq n, j \geq 1
\end{gathered}
$$

is oscillatory if $\{u(i, j)\}$ does not remain positive nor remain negative for $1 \leq i \leq n$ and for all large $j$. In view of Lemma 2.3, we see that if every solution of (2.9) is oscillatory, then so is a solution of the above stated boundary problem. Oscillation criteria for (2.9) can be found in various studies (see for example $[6,7]$ ) and will not be repeated here. Our previous results, however, provides information which go beyond those provided by oscillation theorems. As an example, consider the case of a one dimensional crystal made of $n+2$ atoms of mass $m$. Each atom interacts with its two adjacent neighbors by means of elastic forces with constant $\chi$. The discrete wave equation which describes this model is

$$
\Delta_{2}^{2} u(i, j-1)=\omega^{2} \Delta_{1}^{2} u(i-1, j), \quad 1 \leq i \leq n, j \geq 1
$$

where $\omega^{2}$ is directly proportional to $\chi$ and inversely proportional to $m$. If the first and the last atom are fixed, then the corresponding boundary conditions are given by (4.1).

Recall (see Lemma 2.2) that the eigenvalue problem

$$
\begin{gathered}
\Delta^{2} X(i-1)+\lambda X(i)=0, \quad s \leq i \leq s+J-1 \\
X(s)=0=X(s+J)
\end{gathered}
$$

possesses an eigenvalue

$$
\mu_{J}=4 \sin ^{2}\left(\frac{\pi}{2 J+2}\right)
$$

and the corresponding eigensolution is given by

$$
X(i)= \begin{cases}0 & k=s \text { or } s+J \\ \sin ((i-s+1) \pi /(J+1)) & s \leq i \leq s+J-1\end{cases}
$$


Since $X(i)>0$ for $s \leq i \leq s+N-1$, thus by means of Theorem 3.3, if

$$
\omega^{2} \geq \frac{\mu_{J}}{\mu_{n}}
$$

then no solution of (4.2) can remain positive (or negative) over a rectangle of the form $[1, n] \times[s-1, s+J]$. Since $J$ and hence $\mu_{J}$ can be observed experimentally, our result provides a lower estimate for the unknown $\omega^{2}$ in actual crystals.

\section{References}

[1] S. S. Cheng and R. F. Lu, "Discrete Wirtinger's inequalities and conditions for partial difference equations," Fasciculi Math., 23(1991), 9-24.

[2] S. S. Cheng and B. G. Zhang, "Qualitative theory of partial difference equations (I): Oscillation criteria of nonlinear partial difference equations," Tamkang J. Math., 25(1994), 279-288.

[3] S. S. Cheng, S. L. Xie and B. G. Zhang, "Qualitative theory of partial difference equations (II): Oscillation criteria for direct control systems in several variables," Tamkang J. Math., 26(1995), pp.65-79.

[4] S. S. Cheng, S. L. Xie and B. G. Zhang, "Qualitative theory of partial difference equations (III): Forced oscillations of parabolic type partial difference equations," Tamkang J. Math., 26(1995), pp.177-192.

[5] S. S. Cheng, S. L. Xie and B. G. Zhang, "Qualitative theory of partial difference equations (IV): Forced oscillations of hyperbolic type nonlinear partial difference equations," Tamkang J. Math., 26(1995), pp.337-360.

[6] S. S. Cheng, T. C. Yan and H. J. Li, "Oscillation criteria for second order difference equations," Funkcialaj Ekvacioj, 34(1991), 223-239.

[7] W. G. Kelly and A. Peterson, Difference Equations, Academic Press, 1991.

[8] W. Leighton, "Comparison theorems for linear differential equations of second order," Proc. Amer. Math. Soc., 13(1962), 603-610.

Department of Mathematics, Tsing Hua University, Hsinchu, Taiwan, 30043.

Department of Automation, South China University of Technology, Guangzhou, China 510641.

Department of Applied Mathematics, Ocean University, Qindao, China 266003. 